\title{
Réglages et exploitation de la plaque tournante de Grenoble
}

\author{
The adjustment and operation \\ of the revolving platform at Grenoble
}

\author{
PAR G. CHABERT D'HIËRES \\ INGÉNIEUR DE RECHERGHES DU C.N.R.S. \\ GMEF DE LA SECTION DES MARÉES AUX LABORATOIRES DE MÉCANIQUE DES FLUIDES DE L'E.N.S.E.H.R., GRENOBLE
}

\begin{abstract}
Dans le cadre des études pour l'utilisation énergétique des marées, Electricité de France, la Societe Grenobloise d'Etudes et d'Applications Hydrauliques, le Laboratoire de Mécanique des fluides de l'Université de Grenoble se sont unis pour réaliser une machine destinée à simuler, dans des modèles réduits hydranliques, en plus des parametres classiques, la force de Coriolis due a la rotation de la terre. L'étude et la construction de cette installation ont déjà fait l'objet d'une publication dans La Houille Blanche.

L'exposé qui suit montre la vie de cette machine au cours de ses deux premières années: réglages, mesures des performances, mesures des déformations, construction du premier modele réduit et son tarage.
\end{abstract}

Les marées dans la Manche retiennent l'attention d'un grand nombre de chercheurs. Les ondes y possèdent un caractère bien particulier de par la forme du bassin et influent considérablement sur la géographie humaine et économique des pays qui l'entourent. Les marnages et les courants sont en effet très importants dans une des régions les plus actives du globe.

Les divers moyens que la science met à notre disposition sont employés pour mieux connaître ces courants marins : théories hydrodynamiques, calculs numériques sur modèle mathématique, modèles réduits hydrauliques classiques.

Nous allons décrire les travaux relatifs à l'un de ces derniers : le modèle de la Manche construit sur la plaque tournante de Grenoble.

Sa construction s'est avérée nécessaire pour la mesure de l'influence qu'aurait sur le régime des marées l'utilisation de l'énergie disponible dans le golfe de Saint-Malo et notamment l'influence de l'usine des îles Chausey. De nombreu-

\begin{abstract}
Developed jointly by Electricité de France, SO.GR.E.A.H., and the Fluid Mechanics Laboratory of Grenoble University for a tidal power investigation programme, this machine enables the Coriolis effect due to the earth's rotation to be simulated in hydraulic scale models, in addition to all the various usual parameters considered in this type of work. Its design and construction have been described in a previous article in La Houille Blanche. The first two years of experience with the platform are described, including adjustments, performance and strain measurements, the building of the first scale model, and its calibration.
\end{abstract}

ses recherches ont montré qu'un premier modèle réduit devait avoir pour emprise la Manche entière. Le modèle prenait alors un intérêt géophysique certain; par contre, les difficultés techniques étaient énormes puisqu'il fallait tenir compte de l'effet de la rotation de la terre.

L'Université de Grenoble a décidé la réalisation de sa plaque tournante, machine capable d'entreprendre et de mener à bien de telles études. Elle a bénéficié pour cela du concours d'Electricité de France et de la Société Grenobloise d'Etudes et d'Applications Hydrauliques. Les résultats présentés ici sont le fruit d'une étroite collaboration entre ces trois organismes.

Une description assez détaillée de l'installation a été faite devant la Société Hydrotechnique de France par P. Fontanet lors de la séance du 19 mars 1959. Le mémoire correspondant [1] a été rédigé avec la collaboration de J. Dalverny 
et a paru dans cette revue. Nous pensons qu'il est utile d'en rappeler les lignes essentielles.

Le problème était de faire lourner une masse de l'ordre de 250 tonnes, occupant une surface de $150 \mathrm{~m}^{2}$, autour d'un axe vertical, et cela avec la meilleure précision possible, tant du point de vue de la planéité du mouvement que de la vitesse de rotation. Les tolérances maximales ont été calculées pour permettre l'étude de la Manche. Pour que les seiches dues à un défaut de verticalité de l'axe de rotation ou bien à des déformations périodiques de la plaque, soient inférieures à $2 \mathrm{~mm}$, il fallait, dans le cas de la résonance, que chaque point attaché au fond du modèle reste entre deux plans horizontaux dis- tants de $0,1 \mathrm{~mm}$ à $0,9 \mathrm{~mm}$ suivant les hypothèses.

D'autre part, pour que les seiches dues aux variations périodiques de vitesses de rotation qui entraînent des variations de flèche de la surface d'équilibre de l'eau, soient inférieures à $2 \mathrm{~mm}$, il fallait que cette vitesse soit constante en valeur relative à $0,5 \%$ ou $6 \%$ près suivant les hypothèses.

Notons que ces tolérances, bien que très sévères pour le constructeur, étaient assez larges, puisque l'amplitude des seiches admises atteignait $10 \%$ du marnage à mesurer sur le modèle.

\section{DESCRIPTION DE LA MACHINE}

La figure 1 représente une coupe schématique de l'ouvrage. La dalle $\mathrm{D}$, destinée à supporter les modèles, est en béton armé. Elle a $80 \mathrm{~cm}$ d'épaisseur et est constituée par des hourdis minces de $8 \mathrm{~cm}$ d'épaisseur reliés par un quadrillage de nervures. La vue en plan affecte la forme d'un polygone régulier de 20 côtés inserits dans un cercle de $14 \mathrm{~m}$ de diamètre. Les $2 / 3$ environ de son poids sont supportés au centre par une pivoterie $P$ constituée d'un roulement «'Timken》 et d'une hutée sphérique. Cette charge est transmise au sol par une fondation conique en béton

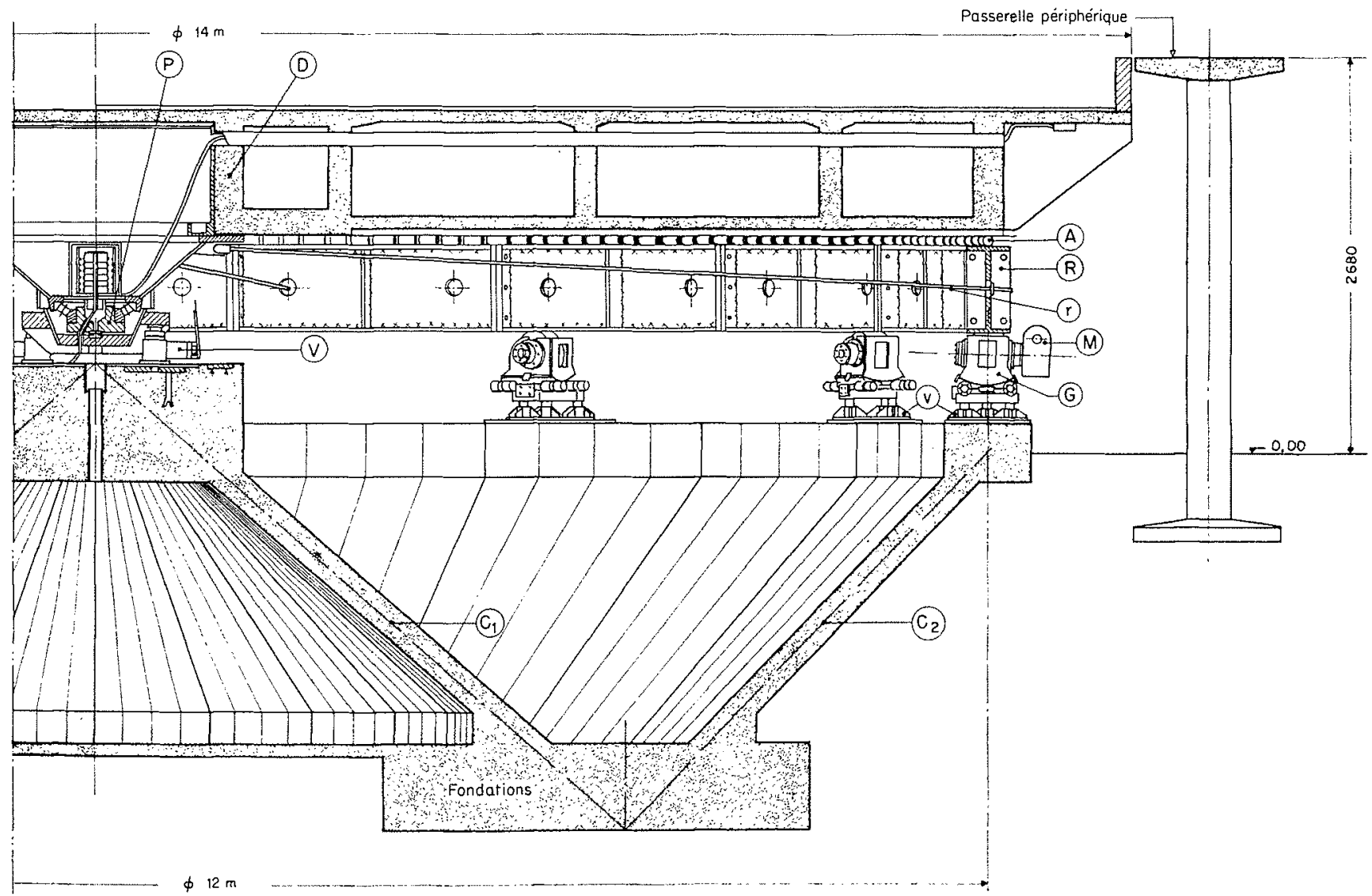

Fri. 1

Demi-coupe schématique de la plaque tournante. 


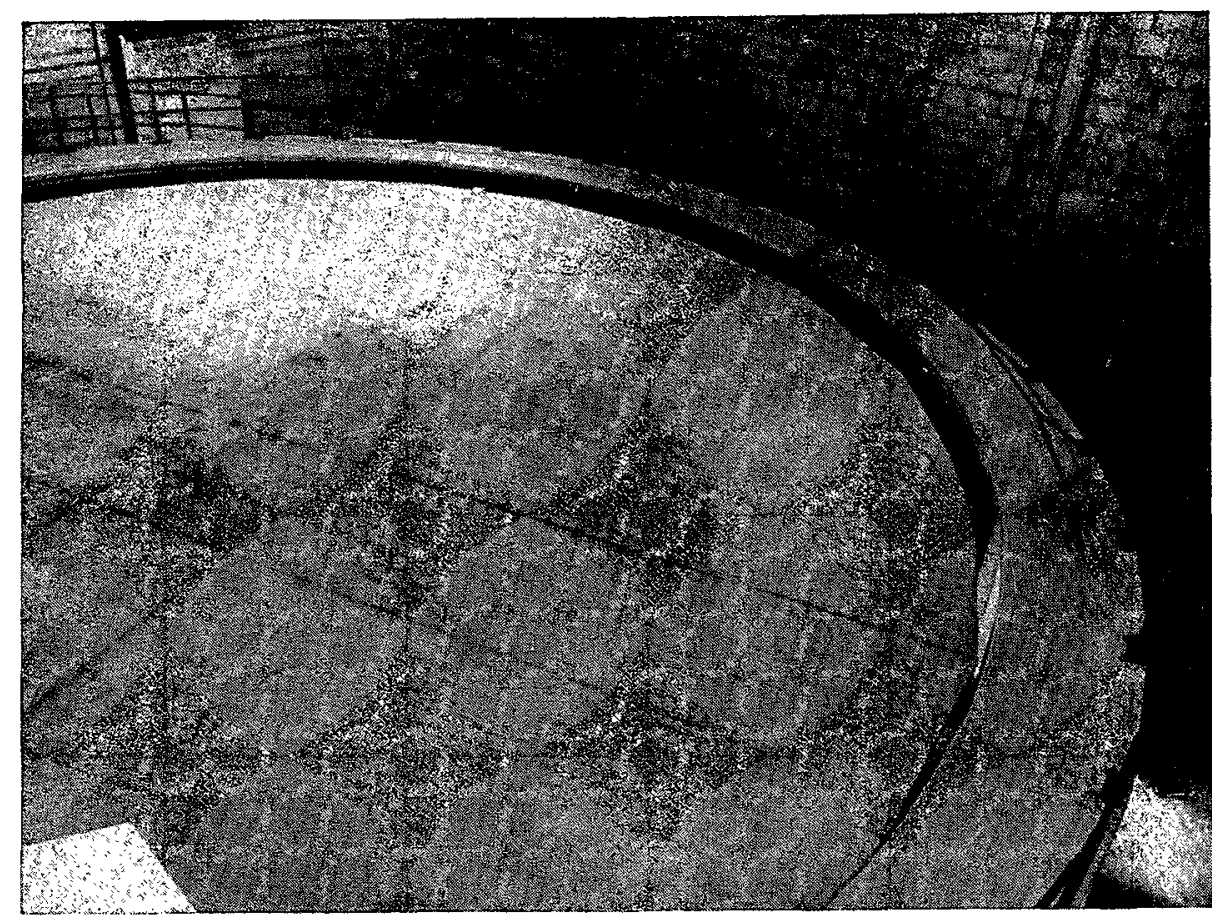

FIG. 2

Plaque tournante vue de dessus avant la construction du modèle.

armé $C_{1}$. Le reste de la charge est porté par 12 galets $G$, dont 6 sont à la fois porteurs et moteurs, répartis sur un cercle de $12 \mathrm{~m}$ de diamètre. Le chemin de roulement est un rail $R$ de $600 \mathrm{~mm}$ de hauteur. Il est lié à la dalle; sa surface inférieure, qui roule sur les galets, et les surfaces de raccordement des vingt éléments dont il est constitué, ont dû être très rigoureusement usinés de façon à matérialiser un plan parfait par lequel le mouvement est guidé. Le rail supporte la dalle par l'intermédiaire de 240 anneaux élastiques A destinés à permettre les dilatations. Chayue anneau est muni de vis, et l'on peut régler la charge qu'il transmet au rail. Ce dernier est lié à la pivoterie par cinq rayons $r$. Les galets transmettent leur charge au sol par l'intermédiaire d'un tronc de cône $\mathrm{C}_{2}$ ouvert vers le haut. La fondation, ainsi constituée par deux surfaces coniques de sens opposés et reliées par une couronne circulaire très épaisse, est fortement armée et forme un bloc très rigide. Les positions relatives des appuis sont ainsi main- tenues constantes, même si l'ensemble vient à basculer du fait de tassements différentiels du sol. La répartition des charges, une fois fixée, doit rester constante.

Les génératrices supérieures des galets peuvent être amenées dans le même plan horizontal grâce à des vérins $v$ placés sous chacun d'eux. D'autres vérins $V$ portent la pivoterie et permettent d'établir la répartition des charges entre les appuis du centre et de la périphérie. Des dynamomètres placés sur chaque galet permettent de contrôler la charge.

Celle-ci doit être suffisante pour assurer l'adhérence des galets moteur's nécessaires à l'entraînement et ne doit pas dépasser la limite imposée par les pièces mécaniques délicates.

Voici quelques ordres de grandeur complémentaires :

- Poids mort de la plaque : 120 tonnes;

- Charge utile de la plaque : 150 tonnes;

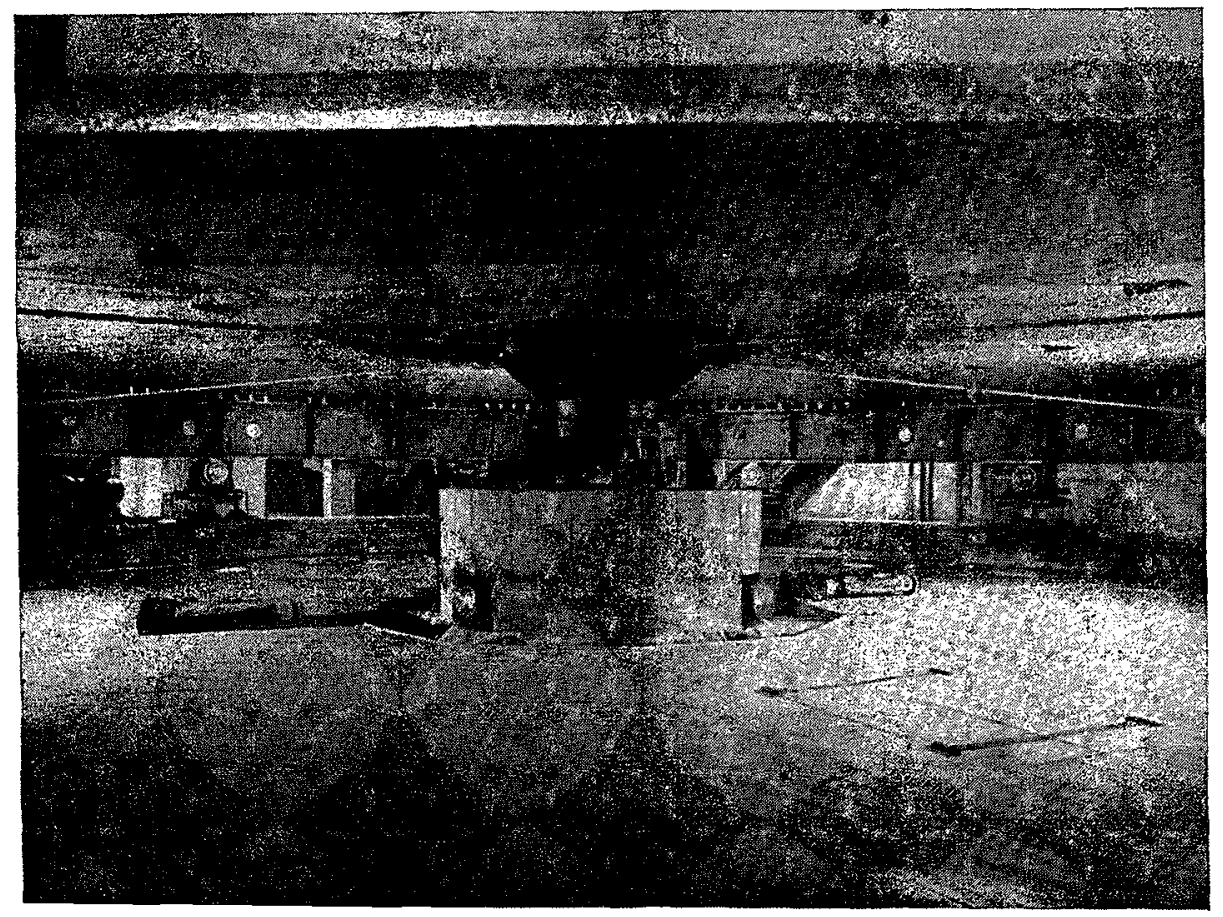

Fig. 3

Plaque tournante vue de dessous. Pivoterie. 
FIG. 4

Vue du rail, d'un galet porteur, et d'un galet porteur et moteur.

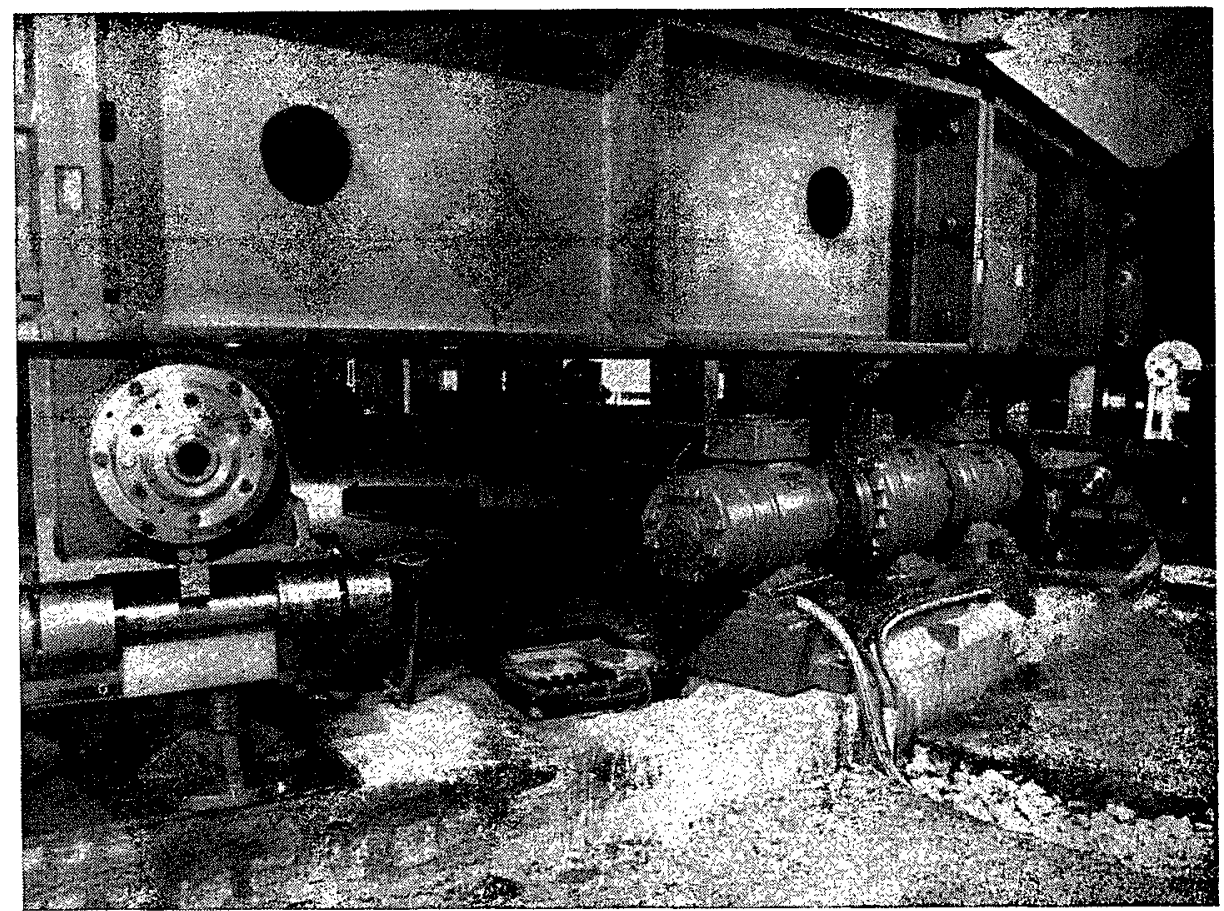

- Charge supportée par le pivot central : 180 tonnes;

- Charge supportée par un galet : $8 \pm 1$ tonne;

- Vitesse de rotation : 0,5 à 2 tours/minute (pourra être portée à 4 tours/minute);

- Diamètre d'un galet : $400 \mathrm{~mm}$;

- Diamètre du roulement Timken : $600 \mathrm{~mm}$.

La rotation est assurée par 6 moteurs $M$ à courant continu, à excitation séparée, dont les induits sont alimentés par deux amplidynes. L'excitation de ces machines est régulée par un courant proportionnel à l'écart de phase qui peut se créer entre un courant à fréquence stabilisée et un, courant fourni par un alternateur multipolaire entraîné par la plaque elle-même.

$\mathrm{La}$ pivoterie, les galets et le rail ont été placés avant le coulage de la dalle proprement dite. Deux mois après le durcissement du béton, la pivoterie a étẻ soulevée pour lui faire prendre tout le poids de la dalle. Le coffrage a été enlevé. Puis, on a baissé l'ensemble pour charger les galets (5 tonnes par galet, c'est-à-dire environ la moitié du poids qu'avait à l'époque la partie tournante). Les galets avaient été, bien entendu, réglés au préalable, c'est-à-dire que leur axe de rotation rencontrait l'axe de la pivoterie et que leurs génératrices supérieures avaient été calées, à l'aide d'un niveau à lunette de précision, dans un même plan. A cette époque alors, ont commencé les réglages proprement dits de la plaque.

\section{REGLAGE DE VERTICALITE DE L'AXE DE ROTATION}

Un long tube horizontal terminé par deux courtes branches verticales, a été placé suivant un diamètre de la plaque. Le tube a été rempli d'eau. Les deux surfaces libres devaient être rigoureusement symétriques par rapport à l'axe de rotation de la plaque pour annuler les effets de variation de force centrifuge (on n'avait pas encore de certitude sur la régulation de la vitesse). Une pointe de mesure «Chatou» permettait d'enregistrer les variations de niveau dans une des branches verticales du tube pendant que la plaque était animée d'un mouvement de rotation lent. Comme les amplitudes étaient faibles, on a pu régler l'appareil de facon à enregistrer avec une amplification de $100^{\circ}$ et déceler ainsi le centième de millimètre. La dérive et les différentes instabilités étaient négligeables durant un essai, pour chacun desquels un étalonnage antérieur et postérieur était fait.
Les dimensions caractéristiques de l'installation sont les suivantes :

- Longueur du tube : $13,5 \mathrm{~m}$;

- Hauteur des pots de mesure : $10 \mathrm{~cm}$;

- Période propre du tube : 5,2 s.

La vitesse de rotation de la plaque devait donc être faible pour que cette période soit petite vis-à-vis de la durée d'un tour. On prenait en général une vitesse de 1 tour en 2 minutes. On a dû freiner le mouvement dans le tube de facon à être voisin de l'amortissement critique.

Avant réglage, les mouvements des surfaces libres étaient de l'ordre de $0,3 \mathrm{~mm}$ et assez désordonnés. Nous avons commencé par redresser le rail en réglant la charge prise par chacun des anneaux sur lesquels repose le béton. Le contrôle de cette opération se faisait en étudiant la 
charge que prenait un galet au cours d'un tour de la plaque. Ce travail a été long et minutieux, mais il a permis d'obtenir une meilleure courbe de variations de niveaux dans le tube en $U$ en fonction de l'angle de rotation de la plaque. Son amplitude était toujours de $0,3 \mathrm{~mm}$, mais elle avait pris la forme régulière d'une sinusoïde. L'axe de rotation était alors bien défini, mais il n'était pas vertical. Il a suffi de calculer d'après cette sinusoïde les quantités dont il fallait monter ou baisser chaque galet et d'effectuer ces corrections pour réduire l'amplitude de cette courbe. En trois approximations successives, cette amplitude a été réduite à $0,03 \mathrm{~mm}$. On n'a pas continué d'améliorer ce réglage parce que les mouvements d'eau dans les tubes devenaient aléatoires et qu'une meilleure précision semblait inutile et illusoire.

D'ailleurs, la machine étant fondée sur un terrain d'alluvions, des tassements différentiels inévitables nous obligent à refaire ce réglage mensuellement. Il n'est pas rare de constater durant ces périodes un basculement de l'ordre de 0,06 à $0,08 \mathrm{~mm}$ sur $13,5 \mathrm{~m}$. A ce propos, nous avons très bien pu déceler l'influence des constructions qui se sont élevées à une trentaine de mètres de la fondation durant ces deux dernières années, l'affaissement de la plaque étant prépondérant du côté le plus éloigné de la construction.

Par contre, nous n'avons pas décelé d'in- fluence notable des variations du niveau de la nappe phréatique qui baigne en quasi-permanence la semelle de la fondation.

Enfin, bien que la plaque soit chargée depuis un an, nous n'avons pas encore constaté de stabilisation dans ces phénomènes de tassement.

Remarque. - On peut objecter à la méthode de mesure au tube en $U$ de la verticalité de l'axe de rotation, le fait que si les mouvements verticaux des deux extrémités du tube sont de même sens, l'appareil ne mesure que la différence de ces mouvements. Nous avons pu montrer que de tels mouvements n'existaient pas en plaçant un tube le long d'un côté du carré inscrit sur la plaque. En arrêtant la plaque dans différentes positions, nous n'avons pas pu déceler des écarts de niveaux supérieurs à $0,02 \mathrm{~mm}$. D'ailleurs, ce même tube a été placé dans de nombreuses positions sur la plaque et on n'a jamais eu d'écarts plus importants. La plaque est donc suffisamment rigide et bien guidée pour ne pas se déformer au cours de sa rotation.

Nous voyons donc que les conditions d'horizontalité ont été satisfaites et que la précision obtenue est même dix fois supérieure à celle qui avait été imposée par les calculs de P. Fontanet. Et l'on a constaté que les seiches dues aux défauts de non planéité du mouvement, peuvent être rendues inférieures à $0,1 \mathrm{~mm}$ dans le modèle de la Manche.
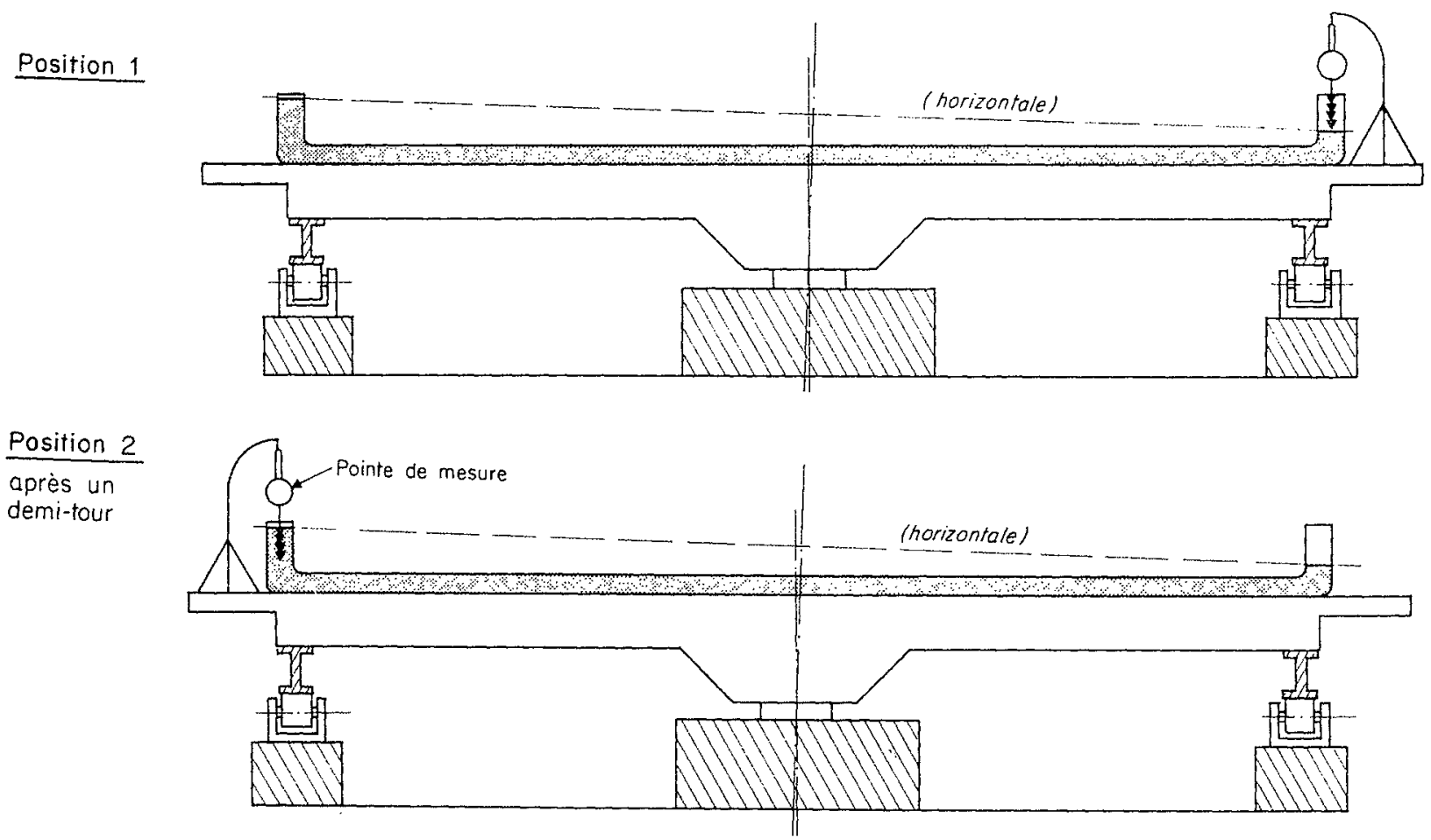

FIG. 5

Schéma du tube en $U$. 


\section{REGLAGE DE LA VITESSE}

Nous ne nous étendrons pas sur le réglage de la vitesse de rotation. Le régulateur, construit par la Société Thomson-Houston, donne satisfaction pour l'exploitation du modèle de la Manche. Nous dirons simplement que l'on a dû parfaire la planéité du rail et le calage des galets en altitude, par une méthode originale, pour diminuer les variations du couple résistant. La vitesse de rotation est maintenue en moyenne constante à 0,0003 près. Les seiches correspondantes sont de l'ordre de $0,1 \mathrm{~mm}$.
Il subsiste toutefois, en certains points du modèle, des ondes courtes à la surface de l'eau. Elles sont dues à certaines vibrations de la plaque créées au passage des jointures du rail sur les galets. Elles sont dues aussi à des accélérations brèves, mais importantes, ordonnées par le régulateur de vitesse. Ces ondes sont créées près des côtes et s'amortissent très rapidement au large et ne gênent pas les mesures. Leur amplitude ne dépasse jamais $0,3 \mathrm{~mm}$.

\section{DESCRIPTION DU MODELLE DE LA MANCHE}

Il est temps de donner une brève description du modèle en signalant les principales sujétions que l'on rencontre sur une plaque tournante.

L'espace disponible est faible et l'emprise du modèle est grande, puisque l'accélération complémentaire n'a d'influence qu'à partir d'aires marines très étendues. L'échelle horizontale est donc petite et l'on est contraint de distordre le modèle pour rendre les amplitudes et vitesses mesurables avec une approximation suffisante d'une part, et surtout pour avoir un écoulement turbulent pendant une fraction importante de la période, à tous les coefficients de marée.

C'est ainsi que l'on a choisi pour le modèle de la Manche $1 / 50000$ en plan et 1/500 en hauteur, ce qui donne pour les temps, une échelle de $1 / 2236$ et $1 / 22,3$ pour les vitesses. La marée semi-diurne a pour période $20 \mathrm{~s}$ et $10 \mathrm{~m}$ d'amplitude sont représentés par $2 \mathrm{~cm}$.

La distorsion de 100 , intéressante du point de vue de l'échelle des temps, l'est beaucoup moins au sujet de la représentation du frottement. La force de frottement est à augmenter considérablement et devient difficilement réalisable. Les études ont été réalisées par la SOGREAH en écoulement permanent; on a commencé par réaliser ce frottement avec de la rugosité de fond, mais le matériau était si épais et si dense que l'on arait deux écoulements superposés, l'un sur les trois quarts de la couche liquide où les vitesses étaient d'un ordre de grandeur convenable, et l'autre sur le quart inférieur où les vitesses étaient très faibles. On a dû répartir le frottement dans la masse. Cette opération est légitime, pourvu que l'on obtienne une répartition des vitesses le long d'une verticalité en similitude avec la nature; c'est-à-dire, pour notre cas particulier, une vitesse constante sur une même verticale. Mais le matériau doit être d'autant plus rugueux que les fonds sont plus faibles. On a essayé, pour avoir le même type de rugosité sur tout le modèle, un grillage dont la densité de maille décroissait de bas en haut. Le grillage choisi donnait un coefficient correct, mais la répartition des vitesses ne pouvait convenir; cette dernière croissait linéairement depuis le fond jusqu'à la surface. La force de Coriolis aurail été fonction de l'altitude, ce qui aurait conduit à des écoulements en spirale. On a dû reprendre un grillage égal sur toute la hauteur de la couche liquide, mais dont la dimension de maille et le diamètre du fil variaient suivant la profondeur d'eau. Le modèle a été divisé en six zones de profondeur différente, à chacune desquelles correspond un grillage déterminé.

Il est à remarquer que la rugosité ainsi calculéc et mesurée pour un écoulement permanent, est un peu trop forte. En effet, on a supposé des frottements quadratiques pendant toute la durée du phénomène. Cette hypothèse est pratiquement réalisée dans la nature, mais pas sur le modèle où l'écoulement est laminaire pendant une fraction non négligeable de la période, 10 à $20 \%$ peut-être dans certaines zones du modèle et même plus tout près des côtes.

D'autres sujétions ont pu être surmontées sans difficulté: la rotation du modèle, par exemple, cntraine une force centrifuge parasite qu'il faut équilibrer en modifiant l'inclinaison des fonds. Il suffit pour cela de prendre comme surface de référence des lignes de niveau, non 


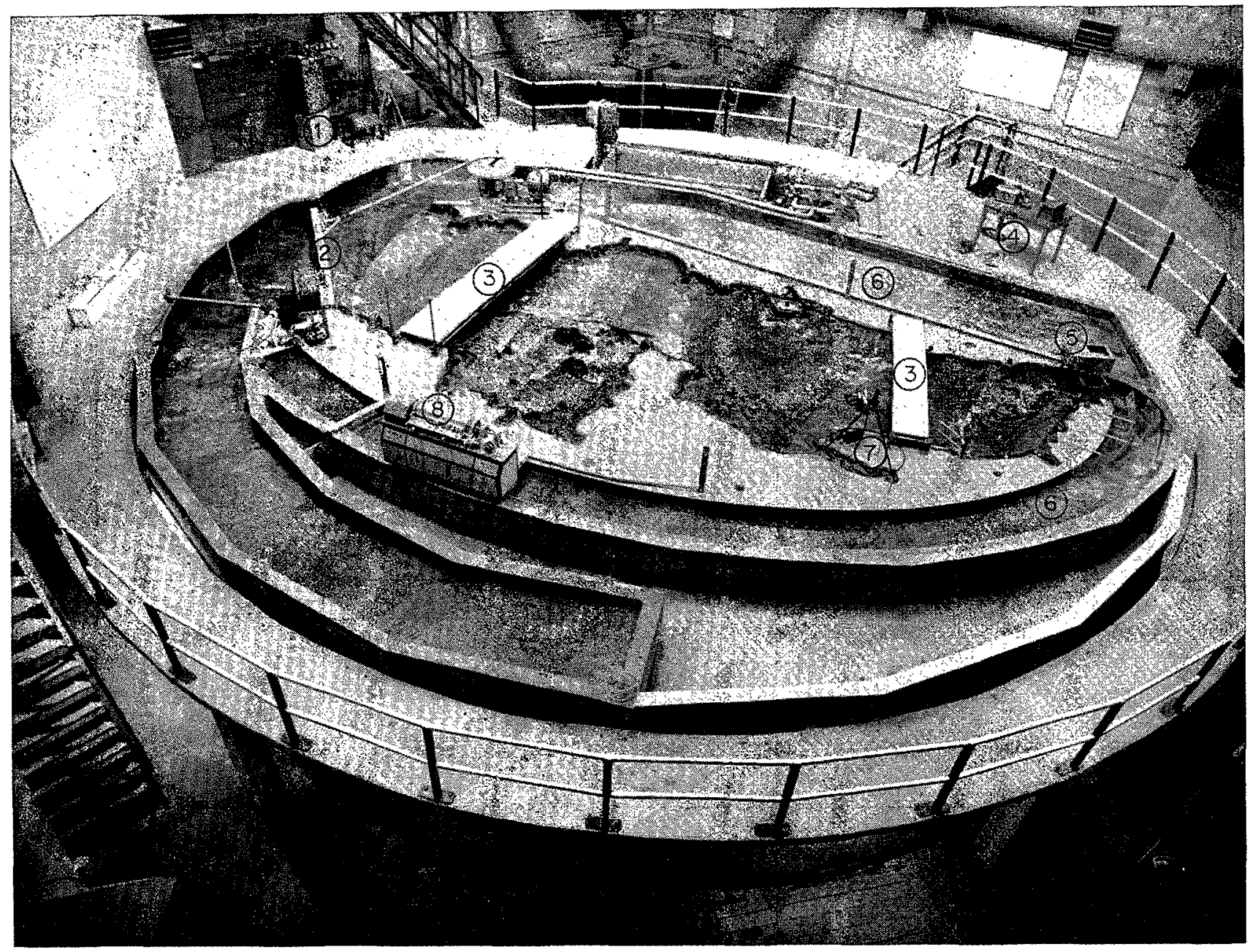

FIG. 6

Vue générale du modèle de la Manche.

1. Régulateur de vitesse de la plaque.

2. Générateur de marée avec son moteur.

3. Passerelles.

4. Appareils pour la mesure de la planéité.
5. Pointe vibrante asservie, type «Chatou».

6. Canaux d'amortissement.

7. Caméra pour la mesure des vitesses.

8. Enregistreur de marées, type «Santon-Marcou». plus un plan horizontal, mais le paraboloïde de révolution qui représente une équipotentielle du champ de forces permanentes qui existe dans le système en rotation.

Enfin, dans le domaine purement technique, on a dû construire le modèle en plusieurs étapes; les charges devaient respecter, grossièrement, il est vrai, l'équilibre statique de la machine. De plus, au cours du chargement, le pivot central devait être soulevé pour lui faire prendre plus de charge que les galets. On a alors vérifié de façon assez satisfaisante les prévisions de fluage de l'ouvrage, les flèches initiales ayant doublé en six mois. Mais il a fallu attendre ce temps-là pour passer la couche de finition qui devait amener les cotes du terrain du modèle à leur juste valeur. Notons que, lors des prochains chargements, ce fluage sera beaucoup moins important.

Les conditions aux limites du modèle ont été étudiées sur modèle mathématique. Le batteur, générateur des marées, est du type «porte». Ce dispositif donne des résultats satisfaisants, puisqu'il schématise assez bien les débits d'énergie entrant dans la Manche; il a aussi l'avantage de laisser une ouverture vers le nord par ou les ondes réfléchies sur les fonds du modèle, ou créées par le fonctionnement d'une usine, peuvent s'échapper et être amorties dans un canal. Ce dernier, ainsi que celui qui représente la mer du Nord, sont du type «quart d'onde». L'étude théorique de tels dispositifs a fait l'objet d'un article publié dans La Houille Blanche par F. Biesel et B. Le Mélhauté [2]. 


\section{QUELQUES RÉSULTATS DES PREMIERS ESSAIS}

Les résultats obtenus après le premier programme d'étalonnage du modèle sont très satisfaisants. Après les retouches faites sur la rugosité comme on l'a déjà signalé, nous avons trouvé les courbes représentatives de la marée dessinées sur les figures 7, 8, 9 et 10 .

Les figures 7 et 8 représentent, le long des côtes, c'est-à-dire aux points où la marée est mesurée, les comparaisons entre le modèle et la nature pour les marées de vives eaux moyennes.
On voit que les courbes sont bien régulières et présentent des écarts systématiques que l'on peut encore espérer réduire en réglant le canal représentant la mer du Nord pour améliorer les marnages à Boulogne, Dieppe et même peut-être à Swanage. En effet, si l'on représente la marée dans cette région par la superposition de deux ondes de Kelvin, l'onde incidente y a une amplitude très faible, l'onde réfléchie une amplitude relativement forte, en opposition de

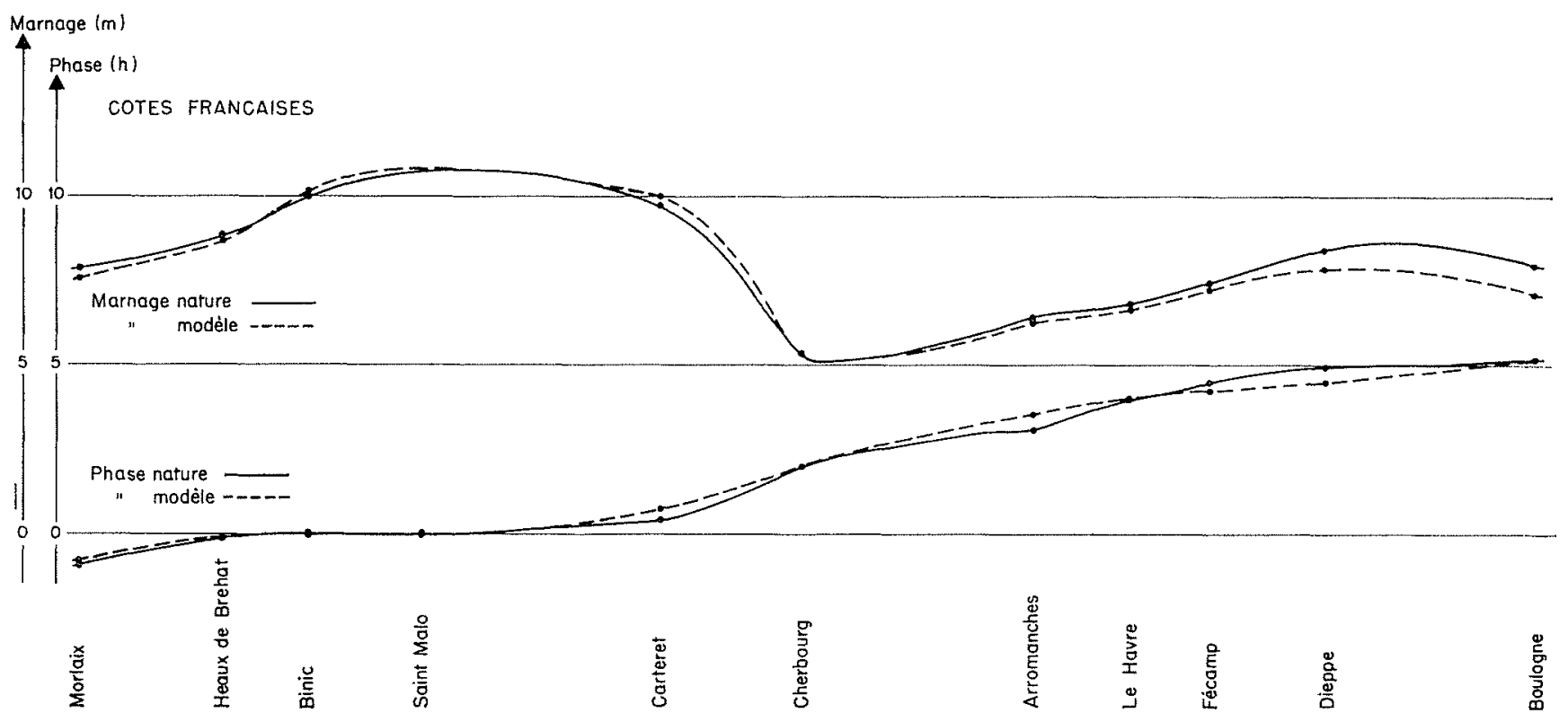

Fig. 7

Marnages et phases de la marée sur les côtes françaises.

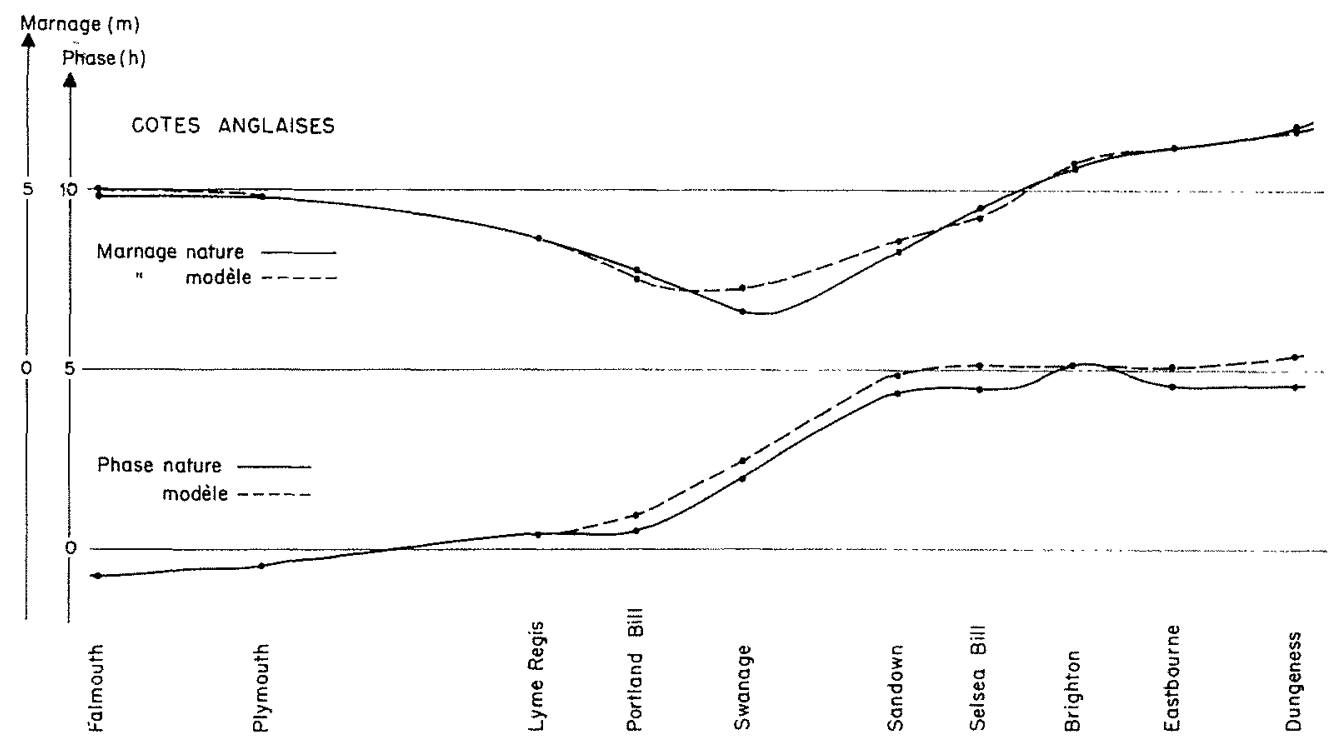

Fia. 8

Marnages et phases de la marée sur les côtes anglaises. 


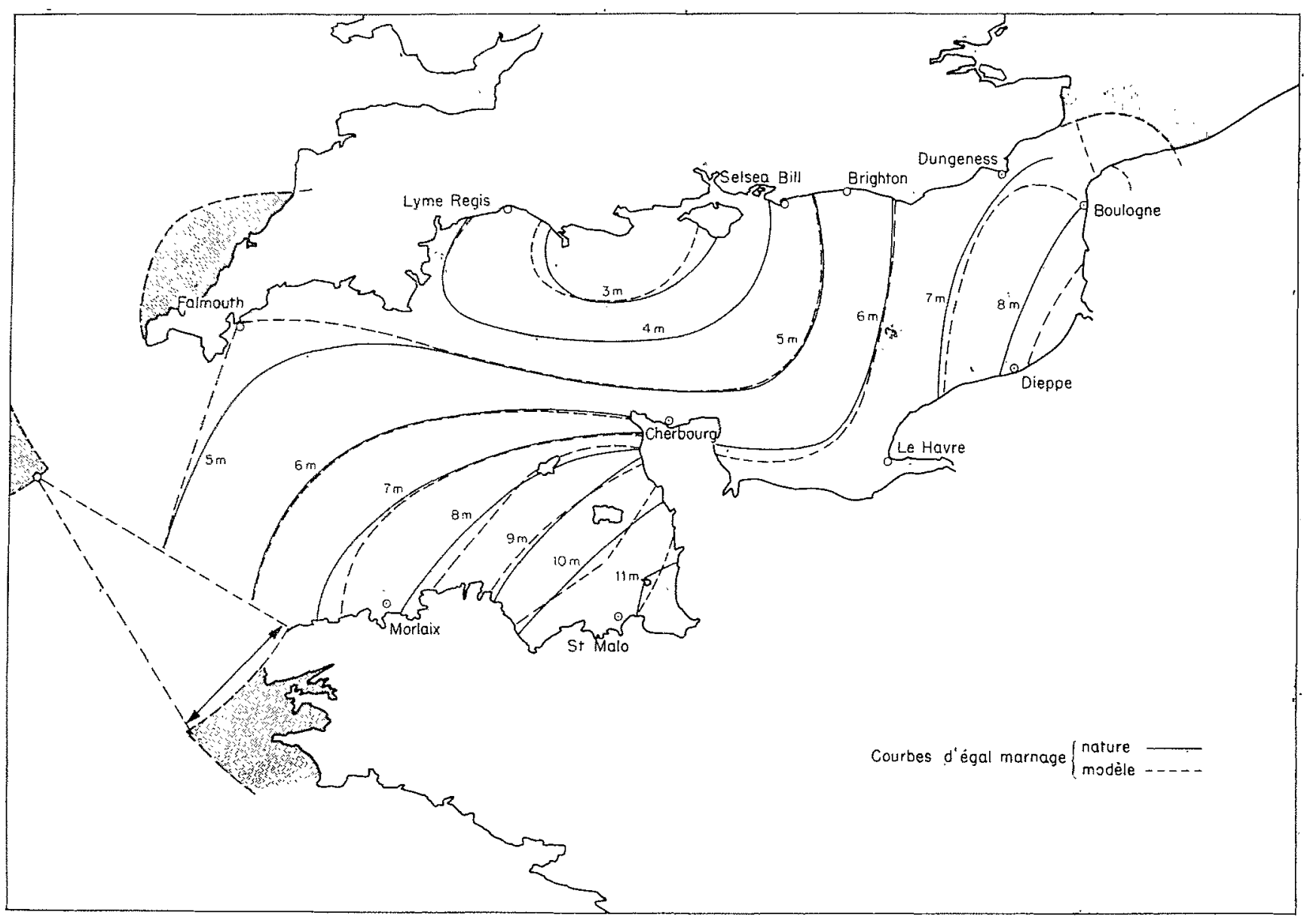

FiG. 9

Lignes d'égal marnage simplifiées.

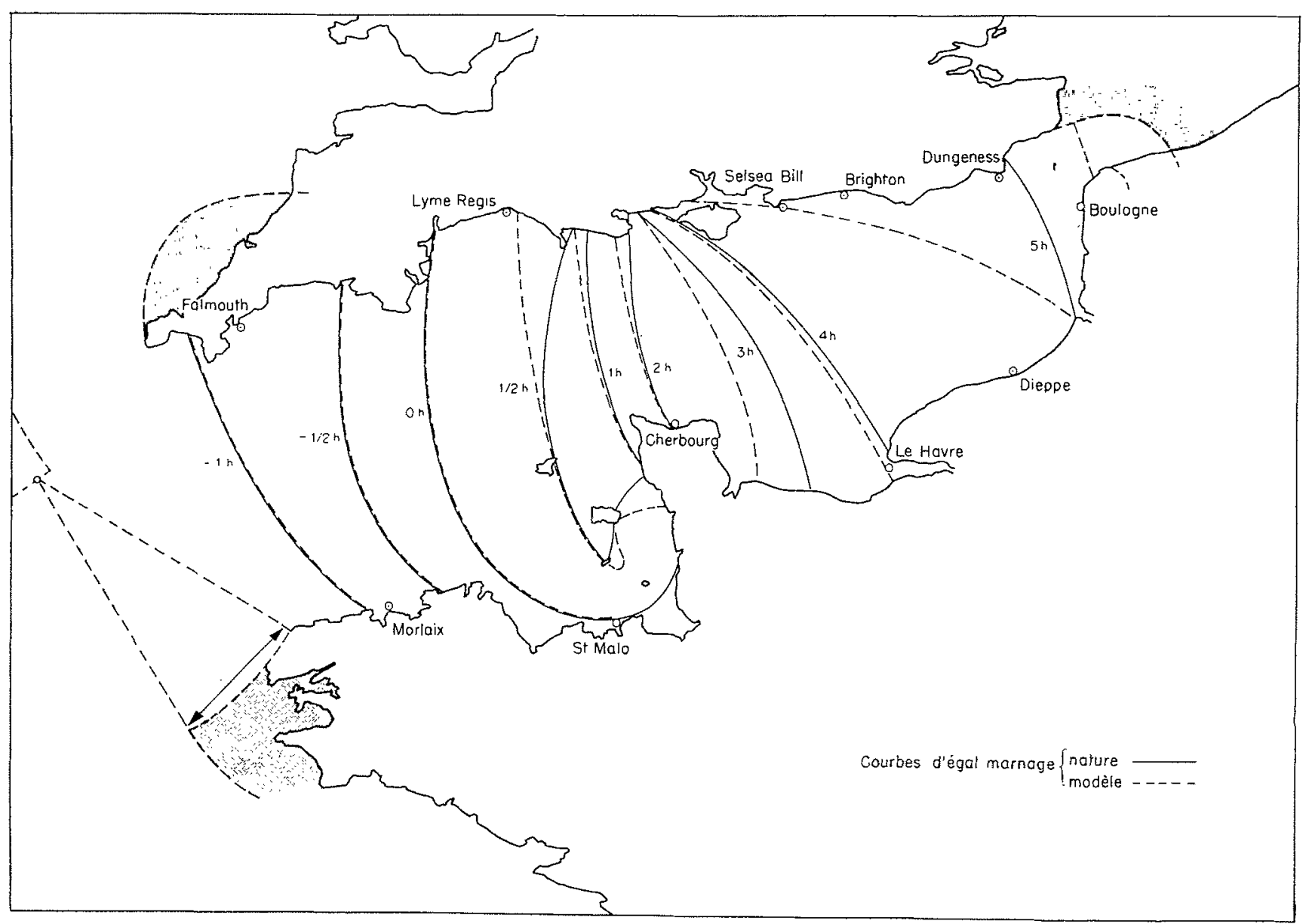

FIG. 10

Cotidales. 
phase avec la première. Une faible erreur sur l'amplitude et la phase de cette dernière, entraîne une grandeur erreur relative sur l'ensemble.

De plus, les marnages semblent encore déficitaires en Manche orientale; ceci tend à prouver que la rugosité est encore légèrement trop forte. C'est d'ailleurs ce que prouvent les graphiques 9 et 10 .

Nous avons essayé de tracer, bien que nous n'ayons pas fait de mesures au large, les lignes caractéristiques de la marée, pour préciser les interventions à pratiquer sur le modèle en vue de parfaire son réglage. Leur enseignement vient confirmer ce que nous avons déjà avancé, notamment en ce qui concerne la rugosité. Sur le réseau des cotidales, le point amphidromique semble être rejeté trop au nord. L'onde réfléchie a donc perdu trop d'énergie. Quoi qu'il en soit, les écarts de marnage sont en moyenne inférieurs à $5 \%$, saử à Dieppe, Boulogne et Swanage, où nous espérons les réduire. Quant aux heures, elles sont bonnes à 30 minutes près et sont encore susceptibles d'améliorations.

Signalons qu'en plus de l'amplitude et de la phase, la forme des courbes représentatives du niveau d'eau en fonction du temps, est satisfaisante (tenue du plein au Havre, écart entre montant et perdant à Saint-Malo, par exemple).

En conclusion, nous pouvons affirmer que le modèle de la Manche est pratiquement réglé et qu'un modèle hydraulique de grandes dimensions, construit sur la plaque tournante de Grenoble, est parfaitement réglable et exploitable. Les problèmes relatifs à leur construction semblent être surmontés et nous espérons que de nouvelles études seront entreprises sur cette machine.

\title{
BIBLIOGRAPHIE
}

(1) P. Fontanet et J. Dalverny. - La Houille Blanche, $\mathrm{n}^{\circ} 5,1959$, p. 598
(2) F. Biesel et B. Le Méhautś - La Honille Blanche, $n^{\circ} 2,1955$, p. 130 .

\section{I C USS I O N}

\author{
Président: M. BanaI
}

M. le Président remercie M. Chabert d'Hrères et, soulignant toutes les précautions que cette première tentative de modèle tournant a nécessitées, se demande si le modèle mathématique ne serait pas plus rentable, dans le cas où on se limiterait à en tirer des courbes d'équimarnage et des courbes d'équi-phases. Il remarque, cependant, que dans quelque temps, on pourra demander à la plaque tournante des informations plus fines et des détails qui échappent au calcul comme, par exemple, de vérifier si la répartition des vitesses sur une verticale est, dans la Manche, vraiment aussi régulière que M. Chabert d'Hières l'indique.

M. CHABEn'T D'Hičres reconnaît que cette répartition est très affectée près de la surface, mais que les mesures faites par M. VOYER avaient donné des répartitions quasi verticales.

M. le Président demande à M. Chabert D'Hières comment, en changeant simplement la maille du grillage,-il a évité l'inconvénient d'avoir une répartition des vitesses triangulaires. M. Chabert D'Hic̀res explique que, dans un premier projet, la maille du grillage a été augmentée avec l'altitude en enlevant un fil sur deux à partir d'une certaine cote, puis un fil sur trois, etc. Cela aurait permis de poser le grillage dans le modèle sans distinction de fond. Mais, ainsi, on s'aperçoit que la rugosité dans les couches supérieures du modèle est nettement inférieure à la rugosité dans les couches infërieures et que, par conséquent, l'écoulement est plus rapide en surface. C'est pourquoi la répartition des vitesses n'est plus sur une verticale.

Actuellement, dans la nouvelle technique, les mailles sont uniformes pour un mêmc fond, mais elles varient avec les fonds.

M. Gougenheim remarque que les observations faites en diverses régions et en particulier, par les Anglais, en mer du Nord et en mer d'Irlande, ne témoignent pas d'une répartition des vitesses de courants de marée suivant une courbe quasi verticale. On a l'habitude de considérer que la distribution affecte la forme d'une parabole, mais d'une parabole dont la plus grande partie est peu inclinée sur la verticale et dont, au contraire, la courbure est très rapide au voisinage du fond. La vitesse diminue lentement à partir de la surface, de sorte que la demi-vitesse est atteinte très près du fond. Sans être absolument uniforme sur toute la profondeur, les courants ne sont pas très éloignés de cette proprićté dans une grande partie de l'épaisseur de l'eau.

D'autre part, M. Gougenheim demande à M. Chabert D'HiĚres, à titre de pure information, si, au cours des mesures de nivellement de la plaque, il n'a pas eu l'occasion de constater des perturbations causées par des secousses sismiques.

M. Chabert d'Hrères répond qu'il n'en a pas constaté, mais que, de toute façon, le système les aurait amorties ou, tout au moins, les aurait cachées. M. Chabert D'Hrènes ajoute qu'il n'a pas, non plus, cherché à savoir si la vitesse d'enfoncement de la plaque était influencée par. les marées terrestres, comme il en a été trouvé au C.E.R.N. à Genève. Des mesures plus rapprochées que la mesure mensuelle pratiquée sur la plaque seraient nécessaires pour constater ces influences et obligeraient à réserver la plaque à ce genre d'essai.

M. de Rouville demande à M. Chabert d'Hières si les divergences plus grandes qu'il a trouvées du côté du Pas-de-Calais ne pourraient pas s'expliquer par le fait qu'autrefois la théorie sur la marée en Manche supposait qu'il $y$ avait deux flots de marée, le plus faible venant du contour des Iles Britanniques par le nord : l'hypothèse de la réflexion sur la côte de picardic a ensuite repris le dessus. Mais ne reste-t-il pas tout de 
même quelque chose de l'ancienne idée, en sorte que cela créerait une perturbation irréelle dans le présent modèle, qui est simplifié, puisqu'il y subsiste senlement la réflexion naturelle sur la côte de picardie.

M. Chabert D'His̀res répond que cela est exact et que le modèle a été réglé en tenant compte de ce paramètre : en faisant varier d'une part la largeur du convergent entre Ie Pas-de-Calais et la cote de Picardie et, d'autre part, la rugosité dans le canal qui représente la mer du Nord, il a pu faire varier le rapport: des amplitudes à Boulogne et à Dieppe.

Mais M. Chaber't D'HiÉnes précise que les essais dont il a parlé sont ceux qui montrent le rapport optimal entre ces deux ports et ne peut donc penser, en améliorant le réglage de ce canal, améliorer encore la répartition des marées dans cette région; il lui semble seulement possible, mais non certain, de modifier un peu la marée de l'île de Wight en changeant la direction des courants dans cette région ou, encore, en changeant légèrcment la phase de l'onde réfléchie, car l'onde incidente a Wight est très faible ct d'autant plus sensible à une très légère modification de l'onde réfléchie.

M. Bonnin rappelle que M. Chatsert D'Hikres a pris la précaution, pour le réglage de la verticalité, de choisir un tube ayant une période très différente de la période de rotation, ce qui donne une bonne précision dans la mesure du décalage. Mais $M$. Bonsin pense que si l'on avait rapproché les deux périodes, ou aurait pu détecter ce décalage avec plus de sensibilité; d'autre part, en utilisant un tube circulaire avec deux tubes de mesure très voisins et de petit diamètre pour diminuer la période, on pourrait détecter un très faible écart sur la vitesse de rotation (effet accélérométrique).

M. Chabert D'Hrères indique que, pour régler la vitesse de rotation, il a monté deux tubes en $U$, l'un suivant un rayon, qui sert de tachymètre, l'autre suivant une corde, qui sert d'accéléromètre. Ce dernier, de $3 \mathrm{~m}$ de long, est extrêmement sensible et décèle des oscillations de l'ordre de $1 / 10^{\circ}$ de $\mathrm{mm}$, parfaitement mesurables à l'aide de la pointe « Chatou».

Dans ces conditions, M. Chabert D'Hières n'a pas éprouvé le besoin d'amplifier le mouvement par résonance, parce que l'on n'aurait jamais pu connaitre de façon précise le coefficient d'amplification du système.

Sur la demande de $M$. le Président, $M$. Bonnefille indique les différences considérables qui existent entre les résultats des modèles fixes et ceux des modèles tournants :

1. Dans ces derniers, les lignes cotidales convergent vers un point amphidromique, d'ailleurs pas assez près de la côte, peut-être à cause d'une exagération de la rugosité;

2“ Sur le modèle de la Manche fixe, les lignes d'équimarnage étaient dans le sens nord-sud au lieu d'être dirigées dans le sens est-ouest; il n'y avait pas de différence entre les marnages des côtes française et anglaise, ni en Manche orientale, ni en Manche occidentale : on observait seulement une augmentation de marnage dans le golfe de Saint-Malo.

M. GovgenHeIM remarque que M. BonNEFILle a parlé des différences entre les résultats acquis sur le modèle tournant de Grenoble et sur le modèle fixe de Chatou. Mais avait-on essayé le modèle de Grenoble en tant que modèle fixe et, si on l'a fait, y a-t-il des différences entre les résultats des deux modèles employés comme modèles fixes? Ce serait un point intéressant à constater.

M. Chabert D'Hières répond que cette vérification est malheureusement très difficile parce qu'on a distordu (courbé) les fonds, mais qu'on pourrait l'étudier grossièrement à la fin du réglage du modèle tournant. En effet, si l'on ne considère que la partie centrale du modèle, c'est-à-dire les deux tiers de celui-ci, la flèche est de l'ordre du centimètre depuis le Heaux-de-Bréhat jusqu'au Havre, alors qu'elle est de $4 \mathrm{~cm}$ si l'on prend la largeur depuis le Pas-de-Calais jusqu'à Brest.

M. Goнin pense que les modèles mathématiques permettent, dans une certaine mesure, d'apprécier les eff ets dus à la force de Coriolis, en comparant les résultats obtenus au cours de deux essais rigoureusement semblables, l'un effectué avec, l'autre sans force de Coriolis. Sur un modèle mathematique, il suffit de modifier une instruction du programme de calcul.

Cette comparaison a été effectuée sur le modèle mathématique de la Manche; les résultats obtenus sont comparables à ceux de $M$. BonNerille et prouvent que les différences de marnage entre les côtes anglaise et française sont principalement dues à la force de Coriolis

M. le Président pense que des différences significatives entre le modèle fixe de Chatou et le modèle tournant de Grenoble arrêté ne pourraient provenir que d'une différence dans la génération de la marée : or, les nombreux essais faits par M. BonNEFille ont montré que les marnages n'étaient guère sensibles à des disposittions quelconques du batteur. Il n'y a donc pas de raison que ces deux modèles soient tellement différents en soi,

M. Gougenhem pense que c'est plutôt dans les rugosités et la répartition des frottements que pourraient exister les causes de ces différences.

M. GoHin ne croit pas que la rugosité ait un caractère artificiel et souligne combien elle est sensible sur la marée. Il rappelle que le professeur Hansen avait utilisé les lois quadratiques et qué, pratiquement, il a utilisé le même coefficient.

M. GougenheIM précíse qu'il ne parle pas du coefficient de rugosité, mais de sa matérialisation sur le modèle : à ce point de vue, les systèmes de grillage lui paraissent avoir une valeur plus efficace que les petites lames de fer utilisées il $y$ a quelques années, mais on a encore recours à des tâtonnements.

M. le Président estime qu'il y a, en effet, une ambiguité lorsque le modèle n'est pas conforme à la nature, par principe, comme c'est le cas du modèle fixe. I rappelle qu'il y avait dans le réglage de M. BonNefiLle une part d'incertitude, puisqu'il réglait un modèle dont on savait très bien qu'il ne pouvait pas réaliser ce qu'il représentait, puisqu'il ne tournait pas. Au contraire, dans le réglage du modèle tournant de $M$. Chabers D'HIÈnEs, il $n^{\prime} y$ a pratiquement qu'une solution : la bonne.

M. Govgenheim est bien d'accord, mais il remarque que l'effet des rugosités matérielles introduites dans le modèle varie avec la vitesse de l'eau, de la même façon que dans la nature, Ior'squ'on ne s'écarte guère d'une valeur moyenne. Mais, pense M. GovGenhem, est-ce que, pour une marée de vive eau et une marée de morte eau, les rugosités soumises à des vitesses très diffèrentes vont se comporter comme dans la nature?

M. Bonnefille rappelle qu'à l'époque où l'on a construit le modèle fixe, il n'avait pas de machines permettant de déterminer par le calcul des rugosités homogènes : actuellement, du fait du calcul préliminaire, la rugosité du modèle tourmant est plus homogène et choque beaucoup moins que celle mise en place sur le modèle fixe.

M. GougenheIm reconnait que le modèle tournant off $r$ plus de facilité à cet égard.

Note De L'AUTEUR. - De nouveaux résultats ont été acquis dans le courant du mois de janvier 1962. On note une nouvelle et très nette amélioration dans la concordance entre modèle et nature. 